\title{
Differentiation Image Quality of MRI Shoulder Joint with Variation RF Coils
}

\author{
Sri Mulyati ${ }^{1, *}$, Luthfi Rusyadi $^{1}$, Ika Mentari Putri Rahmawati $^{2}$, Fatimah ${ }^{1}$, Ardi Soesilo Wibowo ${ }^{1}$, Rini Indrati $^{1}$, and \\ Rasyid $^{1}$ \\ ${ }^{1}$ Poltekkes Kemenkes Semarang \\ ${ }^{2}$ Radiology Technic Diploma, Major of Radiodiagnostic and Radiotherapy, Poltekkes Kemenkes Semarang, Semarang - Indonesia
}

\begin{abstract}
Image Quality of Magnetic Resonance Imaging (MRI) has several factors including SNR dan CNR. Influence Factors of SNR depends on the coil used. MRI shoulder joints in several hospitals can be done with different RF Coil, there are M coil flex and shoulder coil. Are there differences in image quality with MRI shoulder joints between the two coils. This study was conducted to determine differentiation SNR and CNR between them in the coronal proton density sequence of MR shoulder joint. This research was quantitative research with an experimental approach. Scanning was done by using Flex M Coil and shoulder coil with 10 samples. The quality image evaluated of SNR and CNR, data were analyzed using paired t-test with $\alpha=5 \%$. The results of the research showed significant differentiation of image quality between using Flex M Coil and shoulder coil in coronal proton density-weighted of MRI Shoulder Joint with p-value SNR $<0,001$ and CNR $=0,002$. The average of SNR and CNR using shoulder coil is higher than using flex M coil, so the shoulder coil has the better image quality of coronal proton density-weighted MRI shoulder joint.
\end{abstract}

Keywords: Image Quality; MRI Shoulder Joint; Flex M Coil; Shoulder Coil.

\section{Introduction}

The shoulder joint is formed by the humeral head with the glenoid cavity of scapula, this anatomical structure has a wide Range of Movement (ROM), it can allow us to interact with our surroundings [1]. The anatomy of the shoulder joint is ball and socket joint, a cavity at the shoulder joint is very shallow, it makes enable a person to move their arms freely and carry out their daily activities. However, that kind of structure would cause instability of the shoulder joint.

Disruption or problem that occurs on the shoulder joint can be diagnosed by examination of multiple modalities, such as a conventional radiograph and CT scan. However, to reveal the image of a more informative musculoskeletal, MRI examination is better than other modalities. Magnetic Resonance Imaging (MRI) is a diagnostic tool to check and detect parts of the body by using magnetic fields. The results of MRI examination are cross-sectional images of human organs by pieces. MRI uses a magnetic field strength between 0,064 Tesla to 3 Tesla $(1$ Tesla $=1000$ Gauss $)$ and resonant vibrations of the nuclei of hydrogen atoms. Several factors surplus assets, especially its ability to make slices in coronal, sagittal, and axial without manipulating the patient's body position [2].

MRI examination of the shoulder joint is an MRI examination technique with or without contrast media to evaluate the internal disorder of the shoulder joint [3]. There are some weighting used in the MRI of the shoulder joint. Each weighting has different parameters, so it has certain characteristics in assessing a pathological [4]. One of the weighting, which used in MRI examination of the shoulder joint is proton density. Proton density is capable of producing images with high resolution so that the small organ like the superior labrum can be clearly evaluated. In addition, the use of proton density weighting can also be evaluated with good structures that have low signal intensity such as bone or tissue structure, ligaments, and tendons [5]. Proton density, especially in the coronal slice can show overall musculus or the muscles of the shoulder joint propulsion, between the acromioclavicular and glenohumeral joints can be evaluated simultaneously [6].

Several factors that affect the quality of the MRI image include a signal to noise ratio (SNR), contrast-tonoise ratio (CNR), spatial resolution and scan time. SNR is a comparison between the magnitude of the signal amplitude to the amplitude noise (noise) [7]. SNR

\footnotetext{
* Corresponding author: srimulyati@poltekkes-smg.ac.id
} 
greatly affects the image quality, the increase in the value of SNR is followed by an increase in image quality [8]. There are several parameters that affect the SNR, one of them is the use of coil radiofrequency (RF). RF coils are divided into three categories: a transmitter coil, the receiver coil and the transmitter and receiver coils (transceiver coil). Meanwhile, according to the RF coil type, it can be divided into several types including a surface coil, coil volume, phased array coil, solenoidal coil and a Helmholtz coil [7]. According to [9], inhomogeneity of the magnetic field will increase noise and will decrease the value of SNR, so the selection of the right coil for the organ being imaged can increase the value of SNR. In addition, increasing the value of SNR can also be influenced by the type of RF coil structures [10].

Based on observations by the author during fieldwork, MRI examination of the shoulder can be carried out using two types of RF coils ie shoulder coil and flex M coil. Shoulder coil is an RF coil with phased array types, whereas the flex $\mathrm{M}$ coil is an RF coil with a type of surface. In the use of two types of RF coil of this unprecedented study on the image quality of MRI of the shoulder joint. Are there differences in image quality with MRI shoulder joints between the two coils. This study was conducted to determine differentiation SNR and CNR between them in the coronal proton density sequence of the MR shoulder joint.

\section{Method}

This research was a quantitative study with an experimental approach. The study was conducted in the Radiology Installation of Siloam Hospital Karawaci Jakarta. The sample in this study was the image of MRI shoulder joint consisting of 10 samples of MRI images of the shoulder joint using $M$ flex coil and 10 MRI image shoulder joint using shoulder coil. The entire sample was made using the weighting proton density (PD) coronal slice.

The SNR was performed using NessAiver, ie by measuring the object and background noise signals outside the object in the image. SNR measured in SNR tendons, bones and SNR SNR joints. Measured of SNR aided by automated software that exists on a plane MRI, by making the Region of Interest (ROI). ROI on the object supraspinatus tendon is done on the tendon, the head of the humerus bone at the joint and the glenohumeral joint. While ROI noise carried on areas outside of the shoulder joint (see Fig. 1). CNR value calculation is done by subtracting the value of SNR between organs. CNR CNR is calculated that the bonetendon, bone-joint CNR and CNR-tendon joints. Data analyzed using paired t-test with $\alpha=5 \%$.

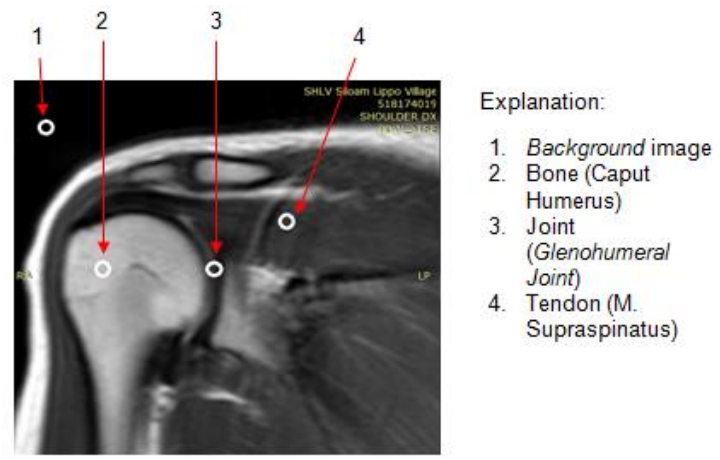

Fig. 1. ROI placement on the measurement of SNR

\section{Results}

The Results of the MRI Shoulder Joint Proton Density Weighted in Coronal Slices

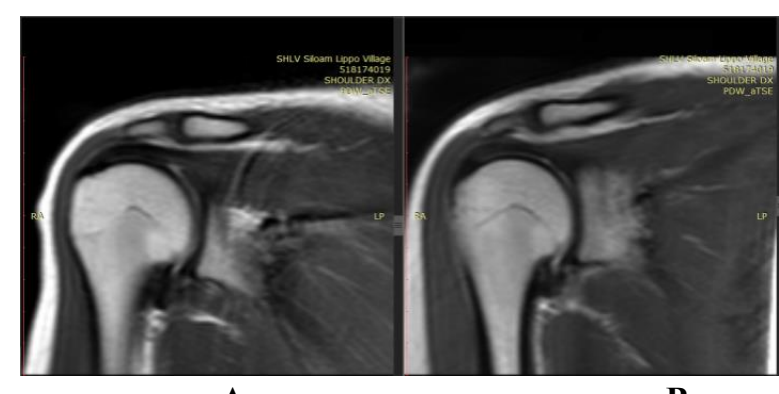

A

B

Fig. 2. A MRI of shoulder joint weighting proton density (PD) coronal slice using shoulder coil. B. MRI of shoulder joint weighting proton density (PD) coronal slice using flex $\mathrm{M}$ coil

1. Image Quality Difference between Using of Flex M Coil and Coil on MRI Shoulder Joint Proton Density Weighted in Coronal Slices.

Normality Test Results Data Values of SNR and CNR on using Flex M Coil and Shoulder Coil SNR and CNR calculation results in the form of a numerical data ratio scale. Normality test data is done by using the Shapiro-Wilk.

Table 1. Results of Normality Test Data of SNR

\begin{tabular}{|c|c|c|}
\hline Variable & $\boldsymbol{\rho}$ Value & Meaning \\
\hline $\begin{array}{c}\text { SNR } \\
\text { Flex } M \\
\text { coil }\end{array}$ & 0.337 & $\begin{array}{c}\text { Normal } \\
\text { Distribution }\end{array}$ \\
\hline $\begin{array}{c}\text { SNR } \\
\text { Shoulder } \\
\text { coil }\end{array}$ & 0.974 & $\begin{array}{c}\text { Normal } \\
\text { Distribution }\end{array}$ \\
\hline
\end{tabular}

From the data normality test results in Table 1, was obtained significance SNR at shoulder coil and flex $M$ coil $>0.05$, so it can be concluded that the data were normally distributed. 
Table 2. Results of Normality Test Data of CNR

\begin{tabular}{|c|c|c|}
\hline Variable & $\boldsymbol{\rho}$ Value & Meaning \\
\hline CNR Flex M Coil & 0.812 & $\begin{array}{c}\text { Normal } \\
\text { Distribution }\end{array}$ \\
\hline $\begin{array}{c}\text { CNR Shoulder } \\
\text { Coil }\end{array}$ & 0.109 & $\begin{array}{c}\text { Normal } \\
\text { Distribution }\end{array}$ \\
\hline
\end{tabular}

From the data normality test results in Table 2, was obtained at the CNR significance shoulder coil and flex $\mathrm{M}$ coil $>0.05$, so it can be concluded that the data were normally distributed.

Results of Paired T-test SNR and CNR on the use of Flex M Coil and Shoulder Coil

After normality test of the data and the results data were normally distributed, subsequent data analysis using paired T-test.

1) Paired T-test of SNR

Statistical test paired T-test on the difference between the use of shoulder coil SNR with M flex coil MR examination of shoulder joint proton density weighted coronal slice obtained the following results:

Table 3. Paired T-test of SNR

\begin{tabular}{|c|c|c|}
\hline Variable & $\boldsymbol{\rho}$ Value & Meaning \\
\hline $\begin{array}{c}\text { SNR Shoulder coil- } \\
\text { SNR Flex } M \text { coil }\end{array}$ & 0.000 & Different \\
\hline
\end{tabular}

Table 3 shows that the SNR values obtained in the use of flex $M$ shoulder coil and shoulder coil have differentiation. This is shown by the results of statistical tests have $\rho$ value / sig. (2tailed) worth 0,000 , which means a smaller or below 0.05 . It can be concluded if the SNR value on the use of shoulder coil and flex M coil MR of shoulder joint proton density weighted coronal slice have significant differences.

2) Paired T-test of CNR

Statistical test paired T-test on the difference between the use of flex M CNR coil by shoulder coil of MR shoulder joint examination proton density weighted coronal slices obtained as follow:

Table 4. Paired T-test of CNR

\begin{tabular}{|c|c|c|}
\hline Variable & $\rho$ Value & Meaning \\
\hline $\begin{array}{l}\text { CNR Shoulder coil- } \\
\text { CNR Flex M coil }\end{array}$ & 0.002 & Different \\
\hline
\end{tabular}

From the data above shown that the CNR values obtained in the use of flex $\mathrm{M}$ shoulder coil and shoulder coil have differentiation. This is shown by the results of statistical tests have $\rho$ value / sig. (2-tailed) worth 0,002 , which means a smaller or below 0.05 . It can be concluded if the value of CNR on the use of shoulder coil and flex M coil MRI of shoulder joint proton density weighted coronal slice have significant differences.

2. RF coil which Better Appears Image Quality between Flex M Coil and Shoulder Coil in MRI of Shoulder Joint Proton Density Weighted Coronal Slices as table 5 below.

Table 5. Image Quality of the Shoulder between Using Flex M Coil and Shoulder Coil

\begin{tabular}{|c|c|c|}
\hline \multirow{2}{*}{ RF Coil } & \multicolumn{2}{|c|}{ Image Quality } \\
\cline { 2 - 3 } & $\begin{array}{c}\text { Average } \\
\text { of SNR }\end{array}$ & $\begin{array}{c}\text { Average } \\
\text { of CNR }\end{array}$ \\
\hline $\begin{array}{c}\text { Shoulder } \\
\text { Coil }\end{array}$ & 114.62 & 66.37 \\
\hline $\begin{array}{c}\text { Flex } M \\
\text { Coil }\end{array}$ & 78.25 & 44.19 \\
\hline
\end{tabular}

From the table 5 we can visualize image quality from different RF Coil in MRI Shoulder Joint Examination like figure 3 below.

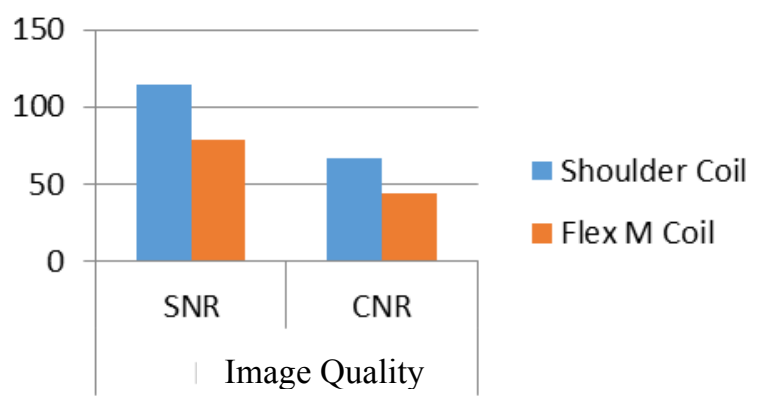

Fig. 3. Graph MRI Image Quality of Shoulder Joint between Flex M Coil and Shoulder Coil

From table 5 and Figure 3 the MRI shoulder joints in coronal slice has difference image quality. Image quality of coil used in MR shoulder joints has SNR and CNR higher of shoulder coil than Flex M Coil.

\section{Discussion}

1. Image Quality Between Flex M Coil and Shoulder Coil of MRI Shoulder Joint Proton Density Weighted in Coronal Slices

Based on research data have been obtained indicate that there are differences in image quality produced between the use of flex M and shoulder coil, from the SNR and CNR produced.

MRI image quality is affected by the 4 aspects of SNR, CNR, spatial resolution and scan time [7]. SNR or Signal to Noise Ratio is the ratio between signal and noise in the MRI image. SNR is influenced by some of the factors, one of which is the use of a radiofrequency coil. While the CNR or the contrast to noise ratio is the difference between organs adjacent to each other, thus 
indirectly CNR value is strongly influenced by the value of SNR.

Statistical test results in SNR values from table 3 have $\rho$ value/sig. (2-tailed) worth 0.000 , which means a smaller or below 0.05. And in table 4. Statistical test results CNR value has $\rho$ value/sig. (2-tailed) worth 0.002 , which means also smaller or below 0.05 . From the results of statistical tests, we can conclude that the results of statistical tests SNR and CNR have $\rho$ value $<0.05$ so that $\mathrm{Ha} \mathrm{Ho}$ accepted and rejected. If $\mathrm{Ha}$ is accepted then no image quality difference between the use of flex $M$ shoulder coil and coil MRI coronal slice of the shoulder joint.

Differences in the quality of the resulting image are due to flexing shoulder $M$ coil and coil are two different types of coils. Flex M coil is a type of surface coil, while the coil is a shoulder type of phased array coil [10]. A surface generally shaped loop coil that is placed close to the object (the signal source) [7]. The advantage of this type of coil is to have a high SNR for imaging superficial structures, because of the proximity to the object and scope only circular area around the coil. Additionally, another advantage of the coil surface is a flexible structure in accordance with the object to be imaged. However, a shortage of this type of coil among others is to have a broad or small area coverage and generates a magnetic field which inhomogenous that causes susceptibility artifacts and the resulting decrease in signal intensity [12].

Phased array coil also called multi-coil which can cover a larger object without causing noise. Phased array coils consist of several surface coils, so that the phased array coil has a high SNR, with a large sensitive area. In the phased array coils, each coil is not interconnected so that SNR is not disturbed. The disadvantages of a phased array coil are the expensive price [13]. Also according to [12], the shoulder coil has a shape surrounding the entire object being examined, so as to produce a homogeneous magnetic field.

Based on the theories mentioned above there was compliance with the research results using statistical methods. There was a significant difference between the image quality of the use of flex M shoulder coil and coil. That is because different types of coils owned both. Flex $M$ coil which was a type of surface coil that can generate high SNR but has a small area coverage and generates a magnetic field which inhomogenous. While shoulder coil was a type of phased array coil that can generate a high SNR, with wide area coverage and produces a rich homogeneous magnetic field.

2. RF coil which Better Appears Image Quality Between Flex M Coil and Shoulder Coil in MRI of Shoulder Joint Proton Density Weighted Coronal Slices

The results of these calculations show that the shoulder coil has a value of SNR and CNR higher than the M flex coil. So, the quality of the image produced on the use of shoulder coil better than the M flex coil. Use of shoulder coil MRI of the shoulder joint is more appropriate when compared to the use of flex $\mathrm{M}$ coil.

Shoulder coil can produce better image quality when viewed from the value of SNR and CNR produced. SNR greatly affect the image quality, the increase in the value of SNR is followed by image enhancement [8]. In addition, the shoulder coil is also an RF coil that is specifically used for the examination of the shoulder joint. The use of radiofrequency (RF) coil corresponding to the object to be inspected is one of the factors that influence the amount of the received signal including the value of SNR [7].
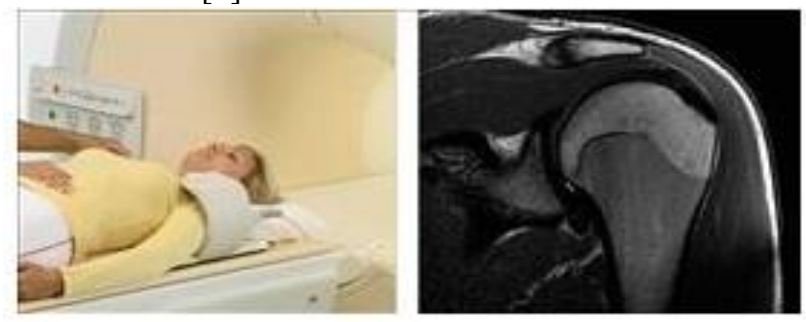

Fig. 4. Applied RF Coil in MRI Shoulder Joint Examination used Shoulder Coil [14]

Offer optimized image quality in all anatomy, because they are: easy to position, compatible with higher SENSE factors, designed for high SNR and optimized image quality, useful for increasing throughput due to scan speed and ease of positioning. Shoulder coil is easy to position. Use the smallest shoulder cup that fits to maximize SNR by the shorter distance to the anatomy [14].
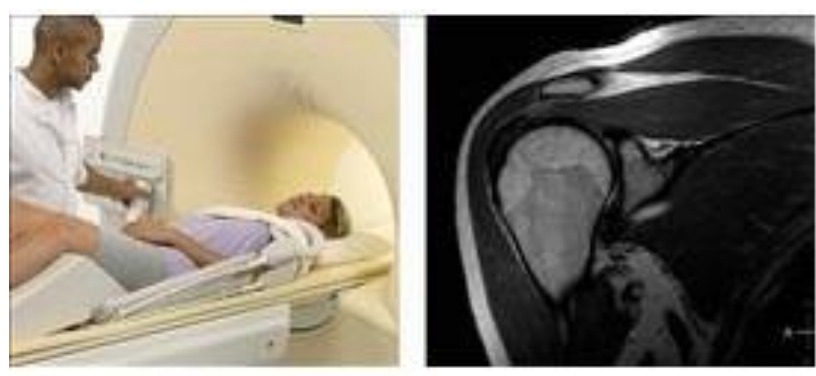

Fig. 5. Applied RF Coil in MRI Shoulder Joint Examination used Flex M Coil [14]

Flex M Coil there are flexible, so they: fit generous-sized patients or patients in pain, can be placed accurately, close to anatomy of interest, which optimizes SNR. The reasons used Flex M Coil: First, position a coil element to cover the deltoid muscle of the shoulder; this element is almost in the sagittal plane. Fix with a strap that also goes through the opening of the second element. Position the second element on top of the first, almost in the coronal plane. Ensure an overlap between coil elements of about $20 \%$ to avoid coupling influencing image quality. Attach both coil elements loosely to the patient by closing the strap [14]. 


\section{Conclusion}

There was a difference in image quality between the use of flex M coil and shoulder coil of MRI shoulder joint proton density-weighted coronal slice with p-value $(\mathrm{SNR})<.001$ and $\mathrm{p}$-value CNR $)=0.002$. Shoulder coil produces better image quality compared to $\mathrm{m}$ flex coil with $\mathrm{SNR}=114.62$ and $\mathrm{CNR}=66.37$.

\section{References}

1. S. Richard Snell, Anatomy Clinic for Medical Students, EGC Jakarta (2000)

2. M. Notosiswoyo, S. Suswati, Utilization of Magnetic Resonance Imaging as a Tools of Patient Diagnosis, Media Litbang Kesehatan XIV, 3 (2004)

3. D. Motamedi, B.M. Everist, S.R. Mahanty, L.S. Steinbach, Pitfalls in Shoulder MRI: Part I-Normal Anatomy and Anatomic Variants, AJR (2014)

4. R. Bitar, et al., MR Pulse Sequences: What Every Radiologist Wants to Know but is Afraid to Ask, RSNA 26, 2 (2006)

5. A. Farber, et al., Magnetic Resonance Imaging Of The Shoulder, Bone \& Joint Surgery 88 (2006)

6. H. Chaudary, S. Aneja, MRI Evaluation of Shoulder Joint : Normal Anatomy \& Pathological Finding A Pictorial Essay And Review, IOSR 2 (2012)

7. C. Westbrook, C.K. Roth, J. Talbot, MRI in Pratice Fourth Edition, Blackwell Science Ltd., United Kingdom (2011)

8. NessAiver, All You Really Need To Know About MRI Physics, University of Maryland Medical Center, USA (1996)

9. D. Weishaupt, et al., How Does MRI Work? An Introduction to the Physics and Function of Magnetic Resnance Imaging, RSNA 246 (2006)

10. K.Y Chen, Development of Radio-Frequency Coil System in Magnetic Resonance Imaging (2012)

11. Philips, Manual Book of MRI Philips Achieva, Philips USA (2008)

12. B. Michael Zlatkin, MRI of the Shoulder, Lippincott Williams \& Wilkins Philadelphia (2003)

13. J. Evert Blink, Basic MRI Physics, support@mriphysics.com, Netherlands (2004)

14. http://clinical.netforum.healthcare.philips.com/us_e n/Operate/Application-Tips/MRI/DedicatedSENSE-coils-and-SENSE-Flex-coils-for-jointimaging accesed 25 July 2019 (2019) 